\title{
Multigroup neutron transport theory in the plane geometry
}

\author{
B.E. Clancy
}

The multigroup form of the transport equation describing the steady state population of neutrons in a plane geometry is studied. The existing techniques of finding numerical solutions to the transport equation in the discrete ordinate approximation are considered in relation to the deep penetration problem of reactor shielding theory. Some of the computational difficulties are examined and a major one found to be the excessive time used in iterating the equation over a large number of mesh points.

A review is given of Chandrasekhar's reflection function method for finding solutions to an astrophysical problem equivalent to the one group form of the deep penetration problem in finite and semi infinite homogeneous media. After generalizing the method it is shown how accurate solutions may be obtained for realistic multigroup problems in semi infinite media without the need for spatial iterations.

For many deep penetration problems in finite heterogeneous slabs only certain integrals of the angular neutron fluxes emerging from the slabs are of interest. A variational principle is devised with the aid of which solutions to appropriately chosen semi infinite medium problems can be used to calculate directly approximations to any integral of these emerging angular fluxes. From the results obtained with a computer progranme embodying these methods it is found that significant savings in computing time can be achieved when a number of survey calculations need to be performed.

Received 3 June 1971. Thesis submitted to Wollongong University College (University of New South Wales), October 1970. Degree approved, May 1971. Supervisor: Professor A. Keane. 
Some possible applications of the reflection function method to other problems in neutron transport theory are examined briefly. 